\title{
Efficient document-image super-resolution using convolutional neural network
}

\author{
RAM KRISHNA PANDEY* and A G RAMAKRISHNAN \\ Department of Electrical Engineering, Indian Institute of Science, Bangalore 560012, India \\ e-mail: rkpandey@ee.iisc.ernet.in
}

MS received 5 April 2017; revised 29 August 2017; accepted 31 August 2017; published online 6 March 2018

\begin{abstract}
Experiments performed by us using optical character recognizers (OCRs) show that the character level accuracy of the OCR reduces significantly with decrease in the spatial resolution of document images. There are real life scenarios, where high-resolution (HR) images are not available, where it is desirable to enhance the resolution of the low-resolution (LR) document image. In this paper, our objective is to construct a HR image, given a single LR binary image. The works reported in the literature mostly deal with superresolution of natural images, whereas we try to overcome the spatial resolution problem in document images. We have trained and obtained a novel convolutional model based on neural networks, which achieves significant improvement in terms of the peak-signal-to-noise ratio (PSNR) of the reconstructed HR images. Using parametric rectified linear units, mean PSNR improvements of 2.32, 4.38, 6.43 and $8.92 \mathrm{~dB}$ have been achieved over those of LR input images of 50, 75, 100 and 150 dots per inch (dpi) resolution and average word level accuracy of almost 43\%, 45\% and 57\% on 75 dpi Tamil, English and Kannada images, respectively.
\end{abstract}

Keywords. Document image; spatial resolution; convolutional neural network; super-resolution; document quality; OCR; PSNR; recognition accuracy.

\section{Introduction}

Very good optical character recognizers (OCRs) have been developed for Tamil and Kannada, using which over 600 books have been digitized and also converted to Braille books, which are being used by blind students $[1,2]$. This contribution, carried out under the banner, Gift of new abilities, resulted in the Manthan award (South Asia and Asia Pacific) in the category of e-inclusion and accessibility in 2014 [3]. The performance of these OCRs on aged documents was subsequently improved by effective segmentation of merged characters [4]. However, the performance is still low on documents scanned at a very low spatial resolution. For example, documents are still being scanned at 75 dots per inch (dpi) in Project Madurai [5] for circulation of the images among the volunteers. The current work specifically aims at improving the quality of the input document [6] so that the OCR is able to obtain better recognition accuracy on low-resolution (LR) document images. We have performed experiments and seen that OCR performance on high-resolution (HR) document images obtained using bi-cubic interpolation is better than that on LR images. However, the problem with this method is that it cannot recover high-frequency components [7]. Further, other methods discussed in the literature for natural images are not suitable for our task. Classical reconstruction-based image

*For correspondence super-resolution (SR) requires multiple LR images with subpixel alignment to reconstruct a HR image [8], whereas singleimage SR methods [8,9], require only one input image. The sparse-coding-based method and its various improvements [10-12] are among the state-of-the-art SR methods. Some recent works that reported on SR of natural images are anchored neighbourhood regression methods [13-15] and self-exemplars [16]. SR based on deep learning is advantageous because almost all aspects of the model are learnt from the data itself. Chao Dong et al [17, 18] proposed natural image SR based on deep learning, which shows that the traditional SR system can be modelled as a convolutional neural network $(\mathrm{CNN})$ for natural images, which achieves state-of-the-art restoration quality and is faster for practical applications than that of Yang et al [10]. Recent work on image SR is based on perceptual loss [19] and generative adversarial network [20]. In [21], the authors have used a weighted combination of mean square error loss, perceptual loss and adversarial loss. Note that all the works have shown their results on natural images. Since we use only binary images for training and testing, we found that this afore-mentioned scheme does not improve the peak-signal-to-noise ratio (PSNR) and OCR accuracy as much as our proposed simple and fast $\mathrm{CNN}$ architecture.

In our work, we have used a basic $\mathrm{CNN}$ with rectified linear unit (ReLU) and parametric rectified linear unit (PReLU) activations, specially trained on binary document images. This uses the concept of shared weight and takes into account the 
Table 1. Three-layer convolutional neural network architecture designed for single document image SR with ReLU or PReLU.

\begin{tabular}{|c|c|c|c|c|c|}
\hline Layer & Type & Filter & Stride & Pad & Output \\
\hline conv1 & $16 \times 16 \times 1$ & $5 \times 5 \times 1 \times 32$ & 1 & NIL & $12 \times 12 \times 32$ \\
\hline Act.fn. & $12 \times 12 \times 32$ & ReLU/PReLU & 1 & NIL & $12 \times 12 \times 32$ \\
\hline conv2 & $12 \times 12 \times 32$ & $1 \times 1 \times 32 \times 16$ & 1 & NIL & $12 \times 12 \times 16$ \\
\hline Act.fn. & $12 \times 12 \times 16$ & ReLU/PReLU & 1 & NIL & $12 \times 12 \times 16$ \\
\hline conv3 & $12 \times 12 \times 16$ & $3 \times 3 \times 16 \times 1$ & 1 & NIL & $10 \times 10 \times 1$ \\
\hline
\end{tabular}

spatial relationship unlike the fully connected network $[22,23]$. We have performed multiple experiments with various parametric settings and different architectures to obtain an effective approach for document image SR.

\section{Problem formulation}

Our objective can be mathematically formulated as follows: given an LR image $L_{i}$, learn a mapping function $F_{\lambda}\left(L_{i}\right)$ while minimizing the loss function $J_{\lambda}\left(F_{\lambda}\left(L_{i}\right), H_{i}\right)$ to obtain a HR image $O_{i}=F_{\lambda}\left(L_{i}\right)$, such that $O_{i}$ has much better PSNR and OCR accuracy than those of $L_{i}$.

We use a parametric CNN model to learn the non-linear mapping function $F_{\lambda}\left(L_{i}\right)$ that minimizes the Frobenius norm between $F_{\lambda}\left(L_{i}\right)$ and the corresponding ground truth HR image $H_{i}$. It is desirable to learn a model/function that fits the training data and generalizes well to the test data. The training set is defined as $I=\left\{\left(L_{i}, H_{i}\right): 1 \leq i \leq N\right\}$, where $N$ is the number of training example pairs, $L_{i}$ is the LR image and $Z_{i} \approx F_{\lambda}\left(L_{i}\right)$ is the output HR image obtained from the CNN. The model is represented as $\lambda=\left\{W_{i}, b_{i}\right\}$, where $W_{i}=\left\{W_{i}^{j}: 1 \leq j \leq n_{i}\right\}$, and $W_{i}^{j}$ is the $j$ th convolution matrix at the $i$ th layer, whose dimensions are listed in table $1, b_{i}$ is a real-valued bias and $n_{i}$ is the number of filters or kernels at the $i$ th convolution layer. The model parameters are learnt using the supervised training algorithm given in algorithm 1 .

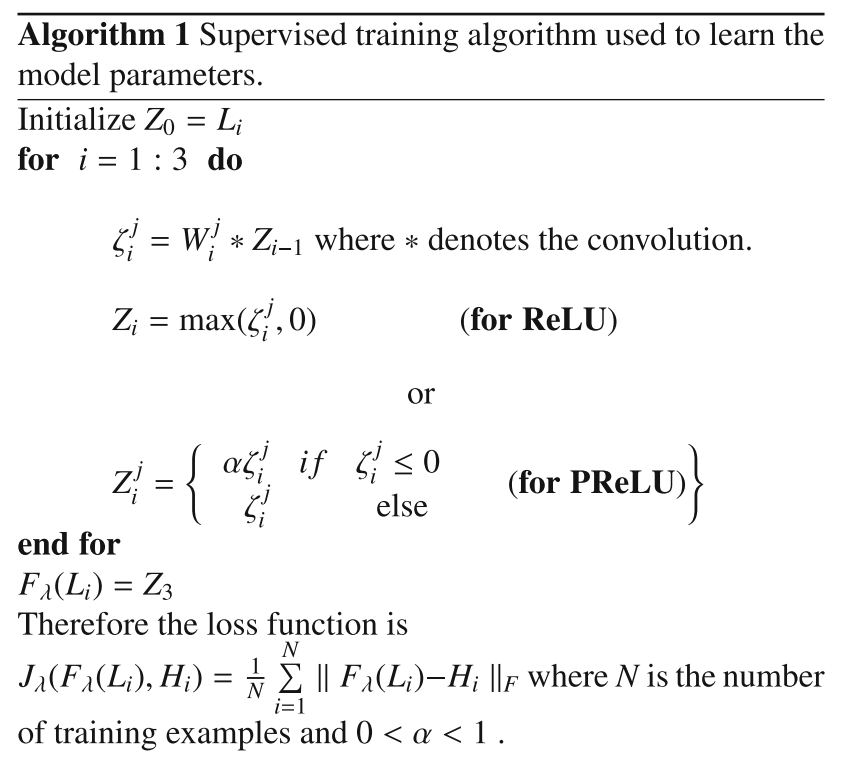

To minimize this loss function with respect to the CNN model parameters, standard back-propagation [24] for gradient computation and momentum-based stochastic gradient descent (SGD) algorithm are used. The use of momentum provides faster convergence with reduced oscillation [25].

\section{Contribution}

Our focus is on solving a real problem of increasing the OCR accuracy on LR document images. To our knowledge, this problem has not been addressed by anyone. Hence, we could not find publicly available standard datasets.

1. We created a large dataset of 5.1 million image pairs specially designed to solve our specific problem.

2. From all the recently available algorithms and techniques, we have tried to select (in terms of test time, higher PSNR and OCR accuracy) the CNN architecture suited for binary document image SR.

3. To make the SR method handle multiple languages and resolutions, we have used a mixture of data from three different languages and resolutions.

4. To show the robustness of the model for language and resolution independence, we have given the results of PSNR improvement and OCR character level accuracy (CLA) and word level accuracy (WLA) for all 3 languages.

5. We report the performance of our enhancement technique in terms of OCR accuracy for Tamil, English and Kannada document images. For Tamil and Kannada, we have used MILE OCR [26] and for English, we have used Google's pytesseract OCR [27]. It is to be noted that the image is enhanced at the pre-processing stage and hence there is no need for any changes in the OCR design.

6. Most of the SR techniques like [13-15, $17,18,28,29]$ are designed for natural images and either do not work well on document images or they are computationally intensive. Our proposed method addresses both the problems and we compare our results with those of one of the famous SR techniques [10]. 


\section{Details of the CNN model employed}

\subsection{Initialization of the network}

Initialization is one of the most important steps while training deep models. If it is not performed with care, the network can become dead or irresponsive and cannot train well. Out of the various initializations proposed in the literature, Xavier initialization [30], is important because it keeps the weights in a fixed range, which takes into account both the fan-in and fan-out of each layer; i.e., $\operatorname{var}\left(W_{i}\right)=$ $2 /\left(n_{o}+n_{i}\right)$. For implementation reasons, it is difficult to find out fan-out. In our work, we have used the initialization technique discussed in [31] $\operatorname{var}\left(W_{i}\right)=2 / n_{i}$.

\subsection{Activation function for the nonlinear layers}

Out of the various activation functions proposed in the literature, we use ReLU [32] and PReLU [31] activations, which give better results for our specific task.

The input to the CNN is an LR image patch of size $16 \times 16$, which, when passed through conv layer 1 containing $n_{1}$ filters, gives an output feature map of size $12 \times 12 \times n_{1}$. This output is then passed on to a non-linear activation function ReLU or PReLU. The number of filters and their sizes are mentioned in table 1 and the model is schematically shown in figure 1 .

The rest of the layers perform the channel-wise convolution on the feature maps of the immediately preceding layers with their corresponding filter kernels $W_{i}$. The output of this convolution operation is then fed to a non-linear activation function. An output image of size $10 \times 10$ is obtained by convolving the feature map with a single filter of size $3 \times 3$. The final reconstructed image is the output of the last convolution layer.

If the input to the convolution layer is of size $w_{1} \times h_{1} \times$ $d_{1}$ and at each layer we have four hyper-parameters, namely the number of filters $n_{f}$, the spatial extent filter $\left(s_{e}\right)$, stride $(s)$ and amount of zero padding $\left(z_{p}\right)$, then the output size is calculated according to the formula [33]:

$\begin{array}{ccl}32 & 16 & 1 \\ \text { Conv filters } & \text { Conv filters } & \text { Conv filter } \\ \text { of size [5 5] } & \text { of size [1 1] } & \text { of size [3 3] }\end{array}$

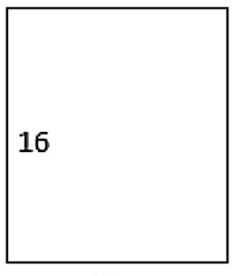

16

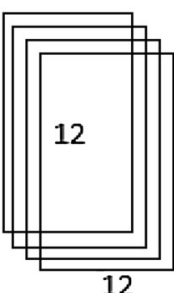

32 feature maps

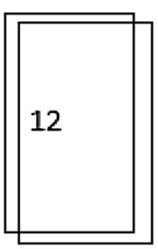

12

16 feature

maps
Figure 1. CNN architecture designed for document image superresolution.

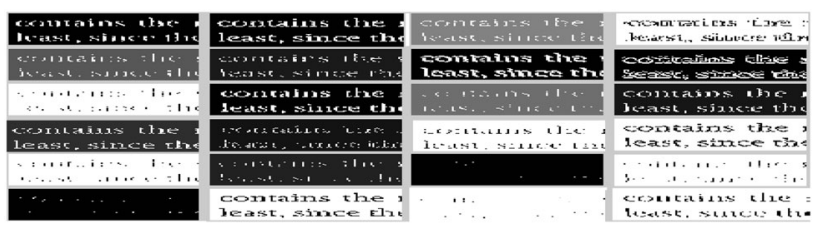

Figure 2. Some responses of the 32 filters of conv1.

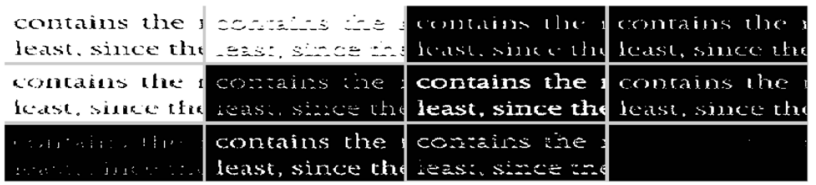

Figure 3. Some responses of the 16 filters of conv2.
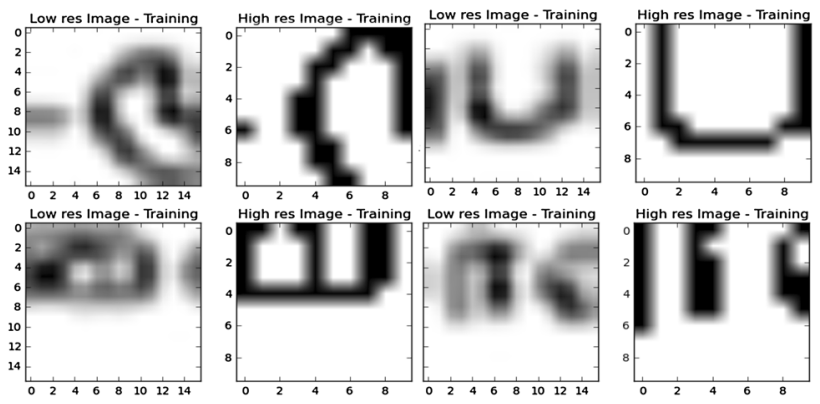

Figure 4. Some LR and HR image patch pairs used for training the CNN.

$$
w_{2}=\left(w_{1}-s_{e}+2 z_{p}\right) / s+1 ; h_{2}=\left(h_{1}-s_{e}+2 z_{p}\right) / s+1
$$
and $d_{2}=n_{f}$

Figures 2 and 3 show the outputs after the actions performed by all the 32 and 16 filters on a binary image at the first and second convolutional layers, respectively.

\subsection{Dataset used for the study}

Since the developed SR technique is intended to work on the three languages and multiple resolutions, we have taken a total of 135 document images for training and 18 for testing English, Kannada and Tamil scanned at three resolutions, namely 100, 200 and 300 dpi. We have randomly selected 5.1 million HR-LR patch pairs from the training images. An LR image is obtained by down-sampling and up-sampling the HR image by a factor of 2 . LR patches are $16 \times 16$ overlapping patches (with horizontal and vertical stride of 6) obtained from the LR image. Intuitively, corresponding $\mathrm{HR}$ patches should be of size $16 \times 16$ taken from the HR image. However, owing to the proposed CNN's architecture and kernal size, the HR patch is actually a $10 \times 10$ patch (and not $16 \times 16$ ) from the HR image. Proper care has been taken in the CNN implementation 


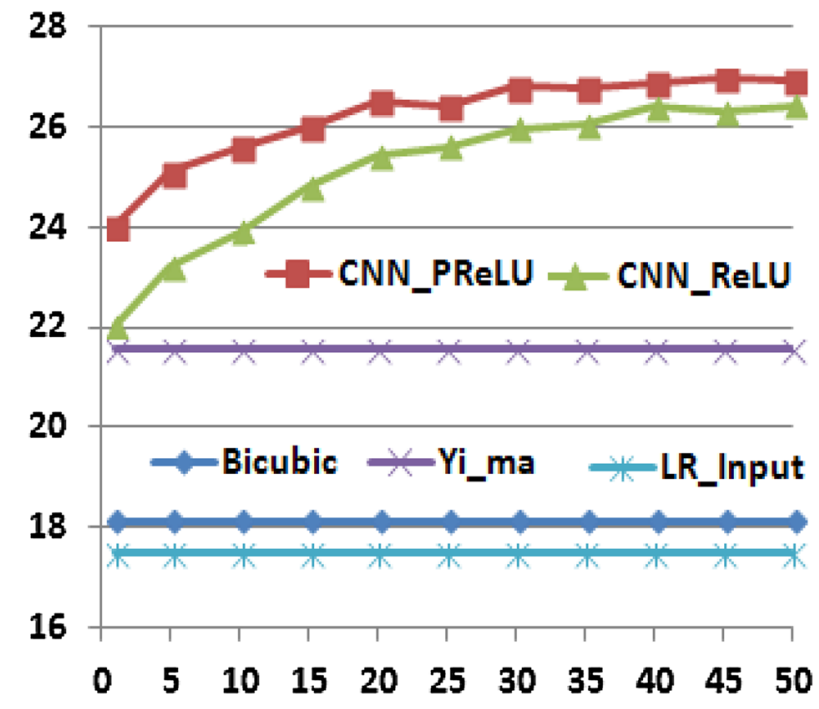

Figure 5. Training curves obtained with ReLU and PReLU activations using 6 test images of $150 \mathrm{dpi}$ : PSNR plotted as a function of training epochs.

Table 2. Average PSNR (in dB) of 18 binary test images (6 each of English, Kannada and Tamil) at different resolutions (Res) in dpi; CNN_ReLU is CR and CNN_PReLU is CPR.

\begin{tabular}{lccccc}
\hline Res & LR_input & Bi-cubic & Yang et al $[10]$ & CR & CPR \\
\hline 50 & 14.57 & 14.94 & 15.05 & $\mathbf{1 6 . 7 4}$ & $\mathbf{1 6 . 8 9}$ \\
75 & 14.98 & 15.53 & 15.83 & $\mathbf{1 9 . 2 4}$ & $\mathbf{1 9 . 3 6}$ \\
100 & 16.60 & 17.37 & 20.22 & $\mathbf{2 2 . 8 3}$ & $\mathbf{2 3 . 0 3}$ \\
150 & 17.48 & 18.20 & 21.55 & $\mathbf{2 5 . 9 6}$ & $\mathbf{2 6 . 4 0}$ \\
\hline
\end{tabular}

such that HR-LR patches correspondance remains intact. Smaller sized image pairs do not perform well. The test dataset is distinct from the training one, to verify that the model obtained generalizes better. Figure 4 shows some sample image pairs from the training set.

\section{Experiments conducted and the results}

We have implemented the model using Theano and Keras $[34,35]$ libraries in python. We used NVIDIA TITAN GTX (12 GB) GPU for training. It is observed that our method is faster than natural-image-based SR [10, 17]. The input training image patches are of size $16 \times 16$. The training dataset is randomly shuffled to ensure that the model does not unnecessarily train to some arbitrary data pattern. We have used the MSE loss function and SGD. We trained our model for 50 epochs with the learning rate of 0.0001 with a constant batch-size of 32 over the epochs.

\subsection{Experimental results}

Suppose the reconstructed image obtained using CNN_ReLU or CNN_PReLU is $O_{i}, H_{i}$ is the ground truth image and the error between the reconstructed image and ground truth is $E_{i}$; then the PSNR, which is used as the metric for image quality, is calculated as follows: $E_{i}=$ $H_{i}-O_{i}$ where $H_{i}, O_{i}, E_{i} \in R^{M \times M}$ and $R M S E=\frac{1}{M}\left\|E_{i}\right\|_{F}$ $\Rightarrow P S N R=20 \log _{10} \frac{255}{R M S E}$.

The OCR accuracy cannot be directly used as the objective function to be maximized. Hence, we have used mean square error as a metric that needs to be minimized and PSNR, and plotted it as a function of the number of training epochs. As long as the PSNR continues to increase (in both ReLU and PReLU cases), we continue training the network for more epochs. We freeze the weights when the PSNR saturates and use it for testing. The training curves in figure 5 are obtained by saving the weights of the model after every 5 epochs of training. The plots show that PReLU activation learns faster than ReLU. Since the accuracy is better in the case of CNN model, we conjecture that representing an image in non-linear manifolds using CNN is better than simple linear models, which can be formulated mathematically as an optimization problem as in [10]. Table 2 shows mean PSNR results for CNN_ReLU and CNN_PReLU methods on English, Tamil and Kannada images scanned at different resolutions. Figure 6 shows one word each of the reconstructed Tamil, English and Kannada HR images, along with their PSNRs.

Table 3 shows the mean CLA and WLA of 6 test images each of Tamil, English and Kannada reconstructed using CNN_PReLU along with those of the LR input images.

\begin{tabular}{|c|c|c|}
\hline அஸாத்ய & contains & గండనైడై \\
\hline அஸாத்ய அஸாத்ய & contair & now: \\
\hline அஸாத்ய & contains contains & 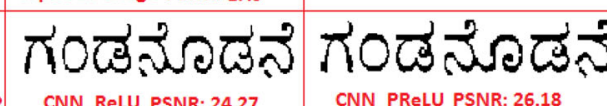 \\
\hline
\end{tabular}

Figure 6. Results with ReLU and PReLU activations on a segment each of Tamil, English and Kannada binary image. 
Table 3. Mean character and word level accuracies for 6 each of Tamil, English and Kannada images at 75 dpi in \%, CPR refers to CNN_PReLU.

\begin{tabular}{lcccccc}
\hline & $\begin{array}{c}\text { LR } \\
\text { input }\end{array}$ & $\begin{array}{c}\text { CPR } \\
\text { input }\end{array}$ & $\begin{array}{c}\text { CPR } \\
\text { Metric } \\
\text { Tamil }\end{array}$ & $\begin{array}{c}\text { Tamil } \\
\text { English }\end{array}$ & $\begin{array}{c}\text { English } \\
\text { Kannada }\end{array}$ & $\begin{array}{c}\text { CPR } \\
\text { Kannada }\end{array}$ \\
\hline CLA & 55.8 & $\mathbf{9 0 . 8}$ & 26 & $\mathbf{7 5 . 1}$ & 66.77 & $\mathbf{9 5 . 1 6}$ \\
WLA & 11.6 & $\mathbf{5 4 . 3}$ & 3 & $\mathbf{4 8}$ & 13.71 & $\mathbf{7 0 . 8 0}$ \\
\hline
\end{tabular}

Table 4. Comparison of mean execution time (MET) of our scheme (CNN_ReLU) with that of Yang et al-sparse representation-based single-image SR [10].

\begin{tabular}{lccc}
\hline $\begin{array}{l}\text { Resolution } \\
\text { (dpi) }\end{array}$ & $\begin{array}{c}\text { CNN_ReLU } \\
\text { MET (s) }\end{array}$ & $\begin{array}{c}\text { Yang et al }[10] \\
\text { MET (s) }\end{array}$ & $\begin{array}{c}\text { Speedup } \\
\text { factor }\end{array}$ \\
\hline 50 & $\mathbf{1 . 5}$ & 125 & $\mathbf{8 3}$ \\
75 & $\mathbf{3 . 3}$ & 282 & $\mathbf{8 5}$ \\
100 & $\mathbf{4}$ & 432 & $\mathbf{1 0 8}$ \\
150 & $\mathbf{1 5}$ & 963 & $\mathbf{6 4}$ \\
\hline
\end{tabular}

\section{Conclusion}

Our CNN-based approach to enhance the quality of document images resulted in mean PSNR improvements of 2.32, 4.38, 6.43 and $8.92 \mathrm{~dB}$ over LR images of 50, 75, 100 and $150 \mathrm{dpi}$, respectively. This improved the OCR CLA and WLA on these images as listed in table 3. Our model scales across 3 languages and is light weight. Scaling across languages is shown by the results on Tamil and Kannada images using MILE OCR [26] and English images using pytesseract OCR (Google) [27], and the proof of light weight is the execution time our model takes to obtain the HR image (table 4). All the input test images and the corresponding HR images are available at this URL: [36].

\section{References}

[1] Aparna K G and Ramakrishnan A G 2002 A complete tamil optical character recognition system. In: Proceedings of the Fifth IAPR Workshop on Document Analysis Systems DAS02, Princeton, NJ, August 19-21, pp. 53-57

[2] Kumar V and Ramakrishnan A G 2002 Machine recognition of printed Kannada text. In: Proceedings of the Fifth IAPR Workshop on Document Analysis Systems DAS-02, August 19-21, Springer, Berlin, pp. 37-48

[3] Manthan award 2014 for the project Gift of new abilities. http:// manthanaward.org/e-inclusion-accessibilty-winner-2014/

[4] Madhavaraj A, Ramakrishnan A G, Shiva Kumar H R and Bhat N 2014 Improved recognition of aged Kannada documents by effective segmentation of merged characters. In: Proceedings of the Tenth International Conference on Signal
Processing and Communication (SPCOM 2014), Bangalore, July 22-24

[5] Project Madurai for ancient Tamil literary works. http:// www.projectmadurai.org/

[6] Pandey R K and Ramakrishnan A G 2017 Language independent single document image super-resolution using CNN for improved recognition. arXiv preprint arXiv:1701.08835

[7] Parker J A, Kenyon R V and Troxel D E 1983 Comparison of interpolating methods for image resampling. IEEE Trans. Med. Imaging 2(1): 31-39

[8] Yang C Y, Ma C and Yang M H 2014 Single-image superresolution: a benchmark. In: Proceedings of the European Conference on Computer Vision. Springer International Publishing, Cham, Switzerland

[9] Nasrollahi K and Moeslund T B 2014 Super-resolution: a comprehensive survey. Machine Vis. Appl. 25(6): 1423-1468

[10] Yang J, Wright J, Huang T S and Ma Y 2010 Image superresolution via sparse representation. IEEE Trans. Image Process. 19(11): 2861-2873

[11] Yang J, Wang Z, Lin Z, Cohen S and Huang T 2012 Coupled dictionary training for image super-resolution. IEEE Trans. Image Process. 21(8): 3467-3478

[12] Yang J, Wright J, Huang T and Ma Y 2008 Image superresolution as sparse representation of raw image patches. In: Proceedings of Computer Vision and Pattern Recognition

[13] Timofte R, Smet V D and Gool L V 2014 A+: adjusted anchored neighborhood regression for fast super-resolution. In: Proceedings of the Asian Conference on Computer Vision. Springer International Publishing, Cham, Switzerland, pp. 111-126

[14] Timofte R, Rothe R and Gool L V 2016 Seven ways to improve example-based single image super resolution. In: Proceedings of the IEEE Conference on Computer Vision and Pattern Recognition

[15] Timofte R, Smet V D and Gool L V 2013 Anchored neighborhood regression for fast example-based super-resolution. In: Proceedings of the IEEE International Conference on Computer Vision

[16] Huang J B, Singh A and Ahuja N 2015 Single image superresolution from transformed self-exemplars. In: Proceedings of the IEEE Conference on Computer Vision and Pattern Recognition

[17] Dong C, Loy C C, He K and Tang X 2014 Learning a deep convolutional network for image super-resolution. In: Proceedings of the European Conference on Computer Vision. Springer International Publishing, Cham, Switzerland, pp. 184-199

[18] Dong C, Loy C C, He K and Tang X 2016 Image superresolution using deep convolutional networks. IEEE Trans. Pattern Anal. Mach. Intell. 38(2): 295-307

[19] Johnson J, Alahi A and Fei-Fei L 2016 Perceptual losses for real-time style transfer and super-resolution. In: Proceedings of the European Conference on Computer Vision. Springer International Publishing, Cham, Switzerland, pp. 694-711

[20] Goodfellow I, Pouget-Abadie J, Mirza M, Xu B, WardeFarley D, Ozair S, Courville A and Bengio Y 2014 Generative adversarial nets. In: Advances in neural information processing systems

[21] Ledig C, Theis L, Huszr F, Caballero J, Cunningham A, Acosta A, Aitken A, Tejani A, Totz J, Wang Z and Shi W 2016 Photo-realistic single image super-resolution using a generative adversarial network. arXiv:1609.04802 
[22] Schmidhuber J 2015 Deep learning in neural networks: an overview. Neural Netw. 61: 85-117

[23] Nielsen M A Neural networks and deep learning. URL: http://neuralnetworksanddeeplearning.com/

[24] Hecht-Nielsen R 1988 Theory of the backpropagation neural network. In: Proceedings of Neural Networks, pp. 445-448

[25] Ruder S 2016 An overview of gradient descent optimization algorithms. arXiv: 1609.04747

[26] Shivakumar H R and Ramakrishnan A G 2013 A tool that converted 200 Tamil books for use by blind students. In: Proceedings of the 12th International Tamil Internet Conference, Kuala Lumpur, Malaysia

[27] Smith R 2007 An overview of the Tesseract OCR engine. In: Proceedings of the Ninth International Conference on Document Analysis and Recognition, ICDAR 2007, IEEE, vol. 2

[28] Wang Z,Liu D, Yang J, Han W and Huang T 2015 Deep networks for image super-resolution with sparse prior. In: Proceedings of the IEEE International Conference on Computer Vision

[29] Kim J, Lee J K and Lee K M 2016 Accurate image superresolution using very deep convolutional networks. In:
Proceedings of the IEEE Conference on Computer Vision and Pattern Recognition

[30] Glorot X and Bengio Y 2010 Understanding the difficulty of training deep feedforward neural networks. In: Proceedings of AISTATS, vol. 9

[31] He K, Zhang X, Ren S and Sun J 2015 Delving deep into rectifiers: surpassing human-level performance on imagenet classification. In: Proceedings the IEEE International Conference on Computer Vision

[32] Nair V and Hinton G E 2010 Rectified linear units improve restricted boltzmann machines. In: Proceedings of the 27th International Conference on Machine Learning

[33] Karpathy A, Li F and Johnson J 2016 CS231n: Convolutional neural network for visual recognition. Online Course

[34] Team, The Theano Development, et al 2016 Theano: a python framework for fast computation of mathematical expressions. arXiv:1605.02688

[35] Chollet F 2015 Keras. https://github.com/fchollet/keras

[36] http://mile.ee.iisc.ernet.in/mile/SR_DocImages.rar 